\title{
English-Language Digital Discourse as Novice Linguocultural and Semiotic Phenomenon
}

\author{
Nataliia Lazebna, Ph.D. \\ Associate Professor, Theory and Practice of Translation Dpt. \\ Zaporizhzhia PolytechnicNational University, \\ Ukraine
}

\begin{abstract}
Changes in taxonomies, onomastics, word groups classification, reterminologization, and other modern linguistic tendencies are related to blurred limits of technological mediation of human activities andthe existence between the virtual and real worlds. 24/7 mediation of human communication using gadgets, on the social media platforms, and using the Internet dominates. Changing focus from social media to basic blocks of digital code within the discourse and correlating these paradigms, it is possible to develop research methodology, which will consider each aspect of English-language digital discourse as one of the most wide-spread communicative spaces. Linguistic, social, and cultural features, as well as a technological aspect of the studied verbal and creative background of human activities, widen the horizon of interdisciplinary studies and integrate effective cooperation of the technorati and the literati.
\end{abstract}

Keywords: digital discourse, cultural aspect, linguistic features, imagery, verbal and non-verbal means.

\section{Cultural underpinnings of tech discourse}

In the global scientific and technical context, it is relevant to focus on the intersection between culture and language. Firstly, from defining these two concepts, outlining their main features and integrating points, the ideas go even deeper and concentrate on specifics of communication between the contemporaries based on their technical background and global background of interaction. There are different aspects of communication between the representatives of different communities, which often do not understand each other, but they should exist in one common global background. The same we can claim about language and cultural connections in the modern technical and scientific discourse. Thus, integrated unity of language and culture is a strong background for the existence of any nation, fostering the ability to go global, and spread its potential worldwide.

Language and culture have often been considered as two inseparable concepts. Currently, in the world of blurred limits, there are many ties between these two concepts, and it is relevant to focus on them in their integration and overlapping. Social migration of people, changes in society are transforming the relationship between culture and language. The names of people represent one of the perfect examples, which highlight the relationship between culture and society. For example, in some cultures, names are important parts of an individual and it is impossible to speak about a wholesome individual without mentioning his or her name. Moreover, names define the destinies of people or predetermine their positive or negative events (at least, Hebrews believe in that) (Danesi, 2016, p. 171).

Moreover, if we cross the limits of technology, we may appeal to our cultural experiences, the mentality of different nations, and the development of a holistic global community. It is possible to refer to Pellegrino Riccardi. After watching his video presentation about crossing cultural limits, there is an option to outline several challenging issues across intercultural communities or societies. He said that the perception of people depends on their cultural background, mentality, traditional representations of one or another phenomenon. Thus, he says that to speak British or American English will make you be positively and respectfully treated abroad.

Pellegrino claims, "We have got different ideas of accepted and familiar" (Pellegrino). For example, when keeping up with people in the queues, people in Norway are more patient and tolerant and try to follow others, while in Italy, for example, where people are more impatient, they see no difference in how to move in queues. In the same way, linguistic features between the Italians and what is the first and foremost in changing and integrating, the language or culture? What is misperception? Where does it come from? There is a myriad of related issues occurring in the related linguistic phenomenon. Moreover, if to focus on the historical background or roots of cross-cultural communication, it is relevant to refer to the basic definitions of culture. For example, "Culture is the characteristics and knowledge of a particular group of people, encompassing language, religion, cuisine, social habits, music and arts" (Zimmermann, 2017). 
The digital literacy of the contemporaries depends on their active involvement in technologies. Thus, in the modern English language, such a tendency is reflected in the accumulation of innovative lexical units, especially in computer discourse. The limits of human existence in the real world and virtual world are blurred and lexemes from generally used English transform into figurative computer terms. For example, "Master/slaveis amodelofcommunicationwhere onedeviceorprocesshasunidirectionalcontroloveroneormoreotherdevices. In some systems, a master is elected from a group of eligible devices, with the other devices acting in the role of slaves" (Master-Slave Technology, Wordspy). The subjective relationship of slaves from the past to their masters are now projecting their semantics on computer terms.

Another borrowing from the African rituals, such as voodoo dolls transformed into "voodoo programming", the computer term, which means the use of programming code written by someone else and which means a complete misunderstanding of the code meaning. These two examples from the English-language computer discourse symbolize that cultural representations common for people are further projected within the technological domain. If to interpret these words, we can refer to comprehending reality and there is a correlation between reality and the virtual world. On the one hand, the world of technological progress is closely related to the cultural and social memories of the nations.

We live in two realities. In real life, we wake up, brush our teeth, and go to work and live our daily routine lives. In the virtual world, we exist and have no obligatory actions and daily drudgery. Gadgets have captured the contemporaries and they cannot get out of this trap. The Internet and social media create a specific level of human existence or transformed reality, which reflects real-life features of the human world.

From the perspective of the 'frontstage' and 'backstage' metaphor developed by Goffman, "the screen becomes our stage where we can choose what to reveal and what to withhold, depending on our perceptions of the audience. As with our real-world (RL) interactions, our identities are constructed through language, interaction, and interpretation" (Discourse in Cyberspace). It means that the visual world enables humans to reconsider their selfidentities.

We can see these language changes. Further, in this paper, we will focus on the anthropocentric expressivity of digital discourse. We will concentrate on anthropic lexical units, visual and graphic elements, which transform digital discourse into an interactive socio-linguistic platform for reflections of human creativity and potential.

\section{Anthropocentric lexical figurative component}

The anthropocentric figurative component is one of the integrative elements of digital discourse. Lexical units used in this type of discourse are similar to common language units. Some semantic features of lexical units can integrate the meaning of expressivity. These lexical units reflect a lively, dynamic image of human experience based on an individual's professional activity. Humans coin every lexical unit of digital discoursebased on their experience (Morris, 1996).

For example, a visual experience of a human produces such units with a figurative component, as cutoff circuit, which means 'an interruption or cessation of a scheme power';flatworm, 'an underlining line', babble 'broadcasting noise'. As far as we can see, the meaning of these units changes in digital discourse. These are not only a chain, a worm, or babbling. These units define objects/activities in the virtual world.

Further, there are two main types of a figurative component in this type of lexical units, - implicit and explicit imagery. We can illustrate implicit imagery in the following way: friends and family virus literary means'a virus of friends and family nature',or a virus, which infects the computer of a user and is sent via email to other users, which are mentioned in the address book of the first infected computer'. Another example illustrates explicit imagery: 9999 bug, which means'a potential problem of computer software, when a program confuses date of ninth of September 1999 (9/9/99) with a figure '9999', or a special code 'the end of a file'.

Acronym NOISE does not mean only 'a sound, especially one that is loud or unpleasant or that causes a disturbance, but it means 'the most furious enemies of Microsoft company: Netscape, Oracle, IBM, Sun, and Everyone else.

Thus, the digital communication area is a transformative power, which changes both human society and language. We cannot underestimate its linguistic features, because they represent changes in our society. The modern English language is vigorously developing through the rapid pace of globalization, which is evident in the lexical structure of social networks.

Communication in digital discourse is dynamic and creative. Analyzed empirical material shows that users (both, professionals and amateurs) develop the vocabulary of English social networks (Facebook, Twitter, LinkedIn, and others). 
We claim that there is a special layer between the real and virtual worlds, which is "mixed reality".Further, we will refer to the concept MIXED REALITY as an anthropic source for the creation of innovative lexical units of computer discourse. It means that users in social media reflect their lives, appearance, actions, and anything else related to their lives. At this point, we can appeal for Marwick and Boyd (p.124), "are we more or less authentic with our book club or gym partner? Whether we are viewed as authentic depends on the definition imposed by the person doing the judging" (Theorizing Identity And Interaction). To answer this question is even more challenging in the virtual world.

From a philosophical perspective, there is a direct relation between anthropology and the Internet. Nowadays, there is a Renaissance of human culture. In other words, an individual projects his lifestyle, activities, self-identity, and other characteristics on the Internet. When we turn on the Internet, we launch the mechanism of coherence between virtual and anthropic realities. The scheme of this mechanism is as follows,

\section{Man $\rightarrow$ mouse button / keyboard $\rightarrow$ sociolinguistic level MIXED REALITY}

As a rule, users transform real data into virtually adapted data and authorize their being in the virtual world. Humans have been improving their writing and reading skills throughout their lives. Nevertheless, human activity on the Internet is voluntary and creative. Educational institutions do not teach individuals the best means to create personal pages on Facebook, write Tweets, or post photos on Instagram. Humans are interested in this type of activity and spend a lot of time on it. Thus, a person lives on both real life and virtual world's levels. Following the existential doctrine of Merlot-Ponty, we can claim that in the virtual world an individual identifies himself differently in comparison with the real world.

Thus, in the virtual world, or on the sociolinguist level MIXED REALITY users describe themselves and coin new lexical units according to their self-identification. According to modern theories in the studied field, "The set of identity features such as our gender, our mother tongue, and our geographical origins are perhaps (more) stable features of the self. These characteristics might be considered the backdrop, or the canvas upon which other more dynamic or temporary identities are placed, such as our profession, our relationships, our membership in special interest groups, etc" (Theorising Identity And Interaction).

In terms of cognitive linguistics, there is a dynamic and anthropological relationship between real and virtual worlds (Loader, 2004).

Further, we will correlate four semantic models used in digital discourse with their real-life prototypes:

1) the static direct anthropic model (digital nomad);

2) the static indirect anthropic model (technoburb);

3 ) the dynamic indirect anthropic model (microblogging);

4) the static non-anthropic model (mouse).

For example, according to American movies, male characters in white hats have always represented good people. The same happens in the digital discourse when white hat hackers correct the mistakes in the software and inform developers about them. «Mell says the attack scripts are posted on hacker Web sites by other hackers, by disgruntled systems administrators trying to draw attention, and eventually patches, to holes in their systems, and by 'white hat' hackersseeking to alert the computer security industry to vulnerabilities». Thus, positive emotional connotation and expressivity are transferred by this lexeme in the English-language digital discourse.

On the contrary, a lexeme dark-side hacker describes a hacker with bad intentions.

«Having a DSL or cable modem service means you have high-speed access to the Internet, but there is a downside. Your computer becomes a tempting target for dark-side hackerswho can more easily break in and steal your banking records, credit card numbers, and passwords».

Another lexical unit, hacktivist, means a hacker, who breaks up the system for further agitation of users. «Members of the Hong Kong Blondes, a covert group, claim to have gotten into Chinese military computers and to have temporarily shut down a communications satellite last year in a 'hacktivist' protest».

Moreover, users project abstract concepts used in real-life in the virtual world. There are many lexemes created via a metaphoric transfer of the concept of DEATH (dead band, dead file, dead space, dead time, deadlock, mortality). Lexemes of this group are united by the following semantic scheme: "the absence of something", "uselessness", "halt", "break", which in general refers to a semantic load of biological concept DEATH. As far as we can see, words with negative emotive semantics prevail in language and speech, because negative aspects of human life are the most memorable for individuals. The same thing happens in the virtual world, when users come across lexemes with a conceptual basis DEATH they experience a negative or pejorative emotional condition.

As far as we can see, anthropic features of humans are projected on lexical units of digital discourse. Individual features or general concepts transfer their meanings from common language into the digital discourse. 


\section{Metaphors in English-language Digital Discourse}

Metaphors in computer texts are characterized by a vividly manifested cognitive nature and metaphoric process modeling, which, in terms of a cognitive metaphor theory depend on the principles of metaphoric processes are based on knowledge interpretation procedures, reflected in cognitive construction-frames (specific unified constructs of knowledge or experience connected by mental reflections) and scenarios (a generalized dynamic experience of human interaction with the world).Foremost, it is possible to define the following source domains in English computer metaphoric term system: 1) anthropomorphic (a human being as a central donor-sphere in metaphoric terms creation); 2) non-anthropomorphic (phenomena and elements of the environment as a donorsphere in metaphoric terms creation). Therefore, an anthropomorphic model of metaphoric terms creation can be represented in the following source domains:

1)biophysical characteristics (deadband,deadfile, deadspace,deadtimeelement, deadlock, diehole, mortality,body, carbonfootprint, crawler, backbonebus);

2)psycho intellectual characteristics (trusttimeout, dumbterminal, don'tcarebit, faultband, troublechart);

3)kinetics(clientpush/pull, handshake, one-touchaccess, flush, bum,burn-in, dike, hack, dieout, to gundown, tobringsystemtoitsknees);

4)food types (applekey, spam, ham,salami (attack), cookie, cracker,menu);

5) attire (cloaking, subnetmask, jacket, bitmask);

6)housing and consumption goods (backdoor, datawarehousing,(chat) room, mailbox, trashcanwindow, filterbox, thread, button,filefolder, clockbus, clockjitter, housekeeping channel);

7) professional gear and actions(crowd, candidatekey, client/serverfilter, hub, stack, companiondescriptor);

8) social status (host, orphans, widow, master, slave, orphan/widowcontrol).

Withthestructureofthe non-anthropomorphicmodelitispossibletodefinethefollowingsource domainsofmetaphoric computertermscreation:

1) landscape (valley, bypass, groundbus)

2) flora and fauna (daisy-chain, betabug, rat'snetprogram, piggyback);

3) natural phenomena (broadcaststorm, flooding, freeze, lightning);

4) sound(doubleclick,babble, barf, wobble);

5) flavor(flavor)

6) image(icon);

7) color (bluescreenofdeath, greenarray);

8) texture of an object (floppydisk, hardcopy, raggedarray, smoothedarray,treechart,slicearchitecture);

9) household goods(inbox, key, diarybug, hash, jacket);

10) means of human communication(datatraffic,messageboard, tracefile, go/no-goevaluation).

Having analyzed anthropomorphic and non-anthropomorphic models of computer terms creation, it is relevant to focus more specifically on the source domain "biophysical characteristics" within the structure of the anthropomorphic model where $50 \%$ of the examples presented in this source are created via the metaphoric transfer of the concept DEATH with explicit involvement of the main lexeme, responsible for the creation of corresponding terms (deadband, deadfile, deadspace,deadtime, deadlock, mortality) thus indicating the dominant anthropomorphic background of English computer terminology.

\section{Visual-graphic elements of digital discourse as media culture semiotics}

Semiotics in media culture is the broad subject area for research. This research may focus on emoticons of social networks (Facebook, Twitter, and others), consider signs and symbols in popular sitcoms (The Big Bang Theory, Community), or trace semiotics in TV news, advertisements, billboards, and in any other sphere of media culture. There is a strong relation between popular culture and mass media. Semiotics within these two spheres is a challenging background for proper consideration.

Particular aspects of this areaare social networks and semiotics in this context.Contemporaries exist on the edge of their real daily lives and existence as users of social media, where they chose the roles they like. A person may differ in real and virtual worlds. Nevertheless, he has an inborn and inherited system of cultural codes and transfers them from his daily life to Internet dwelling. User pictures, signs, symbols, and images reflect his emotional condition, intentions, goals of communication, opinion about anything, and so on.

Signs and symbols, which users of social networks or Internet users transfer from their system of inherited cultural codes, is a fertile ground for potential research. Semiotics of signs and symbols in the web is based on the real experience of a person, his surrounding, habits, feelings, and emotions. 
Roland Barthes was one of the first researchers, who focused on semiotics studying (1988). According to different researchers and scientists, any symbolic system in culture performs the secondary function of another language or text (Durham 2007; Chomsky 2005). At a given stage of development, any symbolic system is holistic and complete. If to penetrate the essence of sign systems in language or visual media, it is possible to analyze, interpret, and comprehend social values and their structure. The ideological background of information, which surrounds people every day, is evident. The ideological background of information is gradually constructed. It is not unintentional and unnatural. There is a different level of people's competence in symbolic meaning and competence. It depends on a person if to encode or decode a given background of signs and symbols. Chomsky conveyed the idea about the deep grammar of signs and symbols (Chomsky 2005). Barthes (1988), Eco (1998), and other scientists considered semiology or semiotics as a system. Multimedia semiotics is a key for the identification of hidden meanings and information background within signs and symbols. People can identify those hidden meanings in the voices of actors, announcers, find them in printed texts, listen to them in music, movies, and Web content.

Every type of mass culture has its code, which is specific to this genre. For example, TV news, a sit-com, or email communication has different types of codes. To be a good email or Internet user, one should be aware of specific nonverbal signs, such as emoticons, smiles, orthographic or punctuation symbols combination, and so on.

Thus, a person is both a sender and receiver of various media culture codes. We distribute different signs and symbols throughout various kinds of media culture. Thus, we convey and interpret the meanings of signs and symbols from one medium to another. These various practices of communication underline that there is a larger global semiotic system. Unfortunately, there is a lack of studies devoted to media culture semiotics. Both, verbal and nonverbal means of communication play the same important role in media culture. We chose the reciprocal background for semiotics research because in social networks users create and use already existent signs and symbols.

Beasley \& Danesi (2002), Durham (2007), Johansen \& Larsen (2002), Carroll (2004), Johansen \& Larsen, S. E. (2002), and Ryan (2005) are current studies devoted to the problems of semiotics in media culture. Moreover, classic works of Barthes (1988), Chomsky (2005), and Eco (1998) are of crucial importance for this research. These scientists developed the essence of semiotics, interpretation of various codes, signs, and symbols. Their works create a strong theoretical background for this study.

Moreover, users of social networks use a system of visual-graphic computer elements. They use iconic signs because with their help they can be sent fully informational and short messages (Morris, 1996). Visual rhetoric means also ensures easiness of information comprehension, the attraction of readers' attention, and even the creation of a comic effect.

For example, let us consider the following answers to one question:

- Will you join me at the party tomorrow?

- "Non."

- "Non >: /"

- "Non :("

Three different answers convey their different meanings. The first answer is neutral, the second is more indignant, and the third express regret and it seems to be the most positive one.

The use of emoticons can play a syntactic role. They can be used after greetings (Hi Jojo :) - as a marker of politeness; in the middle of the message as a means of punctuation, which ends one phrase and starts another one. Emoticons can be independent structural parts of the sentence. For example, :) Yes, I agree with you.

Another graphic feature is to write the same letter several times, or capitalize the whole expression, "AAAH OMG AH JESUSSSS!" "WHATS WRONG!?"Such type of writing means exaggeration or scream.

Therefore, the way of text typing, use of emoticons, and punctuation symbols create another expressive load of digital discourse.

\section{Conclusions}

The expressivity of English-language digital discourse reflects the anthropocentric figurative component as the central one. Lexical units created by users convey features of the human world. The expressive and emotional charge of innovative lexemes transfers semantic features of common language lexical units. Thus, they reflect a lively and dynamic image of the human experience. Visual and graphic means attract the attention of Internet users and diversify English-language digital discourse.

We can go further and refer to other innovative discourses and practices. For example, the discourse of modern technologies, which combine bio-, nanotechnologies, robotics, medicine, and other related fields very often reflect in their terminology cultural glimpses from the past experiences of the nations. 
In terms of anthropology, if to focus on specific features of the innovative technological English-language discourse, figurative terminology reflects the experience of humanity. To popularize the modern discourse, the authors of texts arrange them with imagery terminology, creative stylistic devices, and other techniques.On the one hand, the supporters of classical representatives of knowledge claim that scientific and technical discourse has always been a conservative one, with only one meaning in words, a lack of stylistic means diversifying texts in this discourse, and so on. Very often, the inner form of words and texts is hidden behind the creative imagination of texts authors and recipients' perception.

Language and culture have always been common points for the creation and functioning of nations. These are identifying contours, according to which one nation could be differentiated from another. Blurred limits of modern technological and scientific discourses reflect cultural and social glimpses. People appeal for their common knowledge and project it on innovative technologies. They namenew objects according to their associations, use stylistic devices in the modern scientific and technical discourse to make it more comprehensible for the modern language users. The perspective of the study one can find in focus onthe semioticsof signs and symbols and their classification in English-language digital discourse.

\section{References}

Allen P. (2001). Information Culture is in Need of Development. Network News. Retrieved from: http://www.fundinguniverse.com/company-histories/Microsoft-Corporation-Company-History.html.

Apte, M. (1994). Language in sociocultural context. In: R. E. Asher (Ed.), The Encyclopedia of Language and Linguistics. Vol.4 (pp. 2000-2010). Oxford: Pergamon Press.

Barthes, R.(1988).Mythologies, New York, Noonday Press.

Beasley, R.\& Danesi, M.(2002). Persuasive Signs: The Semiotics of Advertising, New York, Mouton de Gruyter.

Carroll, B.(2004). "Digital Media Revisited: Theoretical and Conceptual Innovation in Digital Domains", Journalism and Mass Communication Quarterly, vol. 81, no. 2, pp. 445+.

Chomsky, N. (2005). Rules and Representations. New York, Columbia University Press.

Danesi, M. (2016). Language, Society and New Media. Routledge.

Durham, F. (2007). "Communication As Perspectives on Theory/50 Ways to Understand Communication: A Guided Tour of Key Ideas and Theorists in Communication, Media, and Culture", Journalism \& Mass Communication Educator, vol. 61, no. 4, pp. 439+.

Eco, U. (1998). Serendipities: Language \& Lunacy (W. Weaver, Trans.), New York, Columbia University Press.

Johansen, J.D.\& Larsen, S.E.(2002). Signs in Use: An Introduction to Semiotics (D. L. Gorlee \& J. Irons, Trans.), London, Routledge.

Loader, B. D. (2004). Cyberprotest: New Media, Citizens, and Social Movements New York: Routledge.

Loeve, S. (2014). Metaphors in Nanomedicine: The Case of Targeted Drug Delivery. Retrievedfrom https://www.academia.edu/8899675/Metaphors_in_Nanomedicine_The_Case_of_Targeted _Drug_Delivery

Morris, M. (1996). The Internet as mass medium. Journal of Computer-Mediated Communication. Retrieved from http://www.asusc.org/jcmc/vol1/issue4/ morris.html.

Preece, J. (2000). Online Communities: Designing Usability, Supporting Sociability. New York : John Wiley \& Sons.

Riccardi, P. The Intercultural Communication. Retrieved from:https://www.youtube.com/watch?v=YMyofREc5Jk

Ryan, M. (2005). "The Media in Question”, Journalism and Mass Communication Quarterly, vol. 82, no. 2, pp. $467+$.

Wordspy. Retrieved from: https://www.wordspy.com/

Zimmerman, K. (July 13, 2017). What is Culture? Retrieved from: https://www.livescience.com/21478-what-isculture-definition-of-culture.html 